\title{
NEUTRON-ANTINEUTRON OSCILLATIONS IN THE TRAPPING BOX
}

\author{
B.O. Kerbikov \\ State Research Center \\ Institute of Theoretical and Experimental Physics, \\ Moscow, Russia
}

\begin{abstract}
We have reexamined the problem of $n-\bar{n}$ oscillations for ultracold neutrons (UCN) confined within a trap. We have shown that the growth of the $\bar{n}$ component with time is to a decent accuracy given by $P(\bar{n})=\varepsilon_{n \bar{n}}^{2} t_{L} t$, where $\varepsilon_{n \bar{n}}$ is the mixing parameter, $t_{L} \sim 1$ sec in the neutron propagation time between subsequent collisions with the trap walls. Possible corrections to this law and open questions are discussed.
\end{abstract}

\section{Introduction}

During several decades the problem of nucleon instability is a subject of intense and diversified theoretical and experimental studies. An interesting facet of this fundamental problem is a hypothetical process of neutronantineutron oscillations [1]. Such oscillations have been thoroughly discussed in free-space regime and inside nuclei -see e.g. [1]- 15] and references therein. The third and very interesting possibility to search for $n-\bar{n}$ oscillations is to use ultra-cold neutrons (UCN) confined in a trap. This subject was discussed by several authors [2, 3, 6] but in contrast to the first two regimes the picture of $n-\bar{n}$ oscillations of UCN remains rather obscure. On the other hand several experiments of this kind are in preparation now. Therefore it is appropriate to address this subject again. We shall follow two complementary lines of arguments. The first one is based on simple qualitative 
estimates while the second makes use of the time evolution equation focusing on the interaction of the two-component $n-\bar{n}$ system with the walls of the trap. Both approaches lead to a conclusion that the $\bar{n}$ component grows (approximately) linearly with observation time contrary to quadratic time dependence in the free-space regime.

\section{Relevant Parameters}

We start by introducing a set of definitions and parameters. Our treatment will be somewhat schematic in the sense that we do not consider any specific geometry of the trap, concrete UCN spectrum, and a variety of the trap materials. All these points can be easily accounted for as soon as one sticks to a given experimental setup.

First we remind that neutrons with energy $E<10^{-7} \mathrm{eV}=100 \mathrm{neV}$ are called ultra-cold. A useful relation connecting the neutron velocity $v$ in $\mathrm{cm} / \mathrm{sec}$ and $E$ in neV reads

$$
v(\mathrm{~cm} / \mathrm{sec}) \simeq 10^{2}\{E(\mathrm{neV}) / 5.22\}^{1 / 2} .
$$

In particular the velocity corresponding to $E=100 \mathrm{neV}$ is $v \simeq 4.4 \cdot 10^{2} \mathrm{~cm} / \mathrm{sec}$.

A less formal definition of UCN involves a notion of the real part of the optical potential corresponding to the trap material. Namely, neutrons with energies less than the height of this potential are called ultra-cold. The two definitions are essentially equivalent since for most materials the optical potential is of the order of $100 \mathrm{neV}$ (see below).

Our main interest concerns strongly absorptive interaction of antineutron component with the trap wall. Therefore very weak absorption of neutrons on the wall will be neglected. Interesting by itself this problem is out of the scope of the present work. According to the second definition of UCN (i.e. $\left.E<U_{n A}\right)$ they undergo complete reflection from the trap walls and may be stored for about $10^{3} \mathrm{sec}(\beta$ - decay time) as was first point out by Ya.B.Zeldovich [7]. For each material the limiting neutron velocity is given by (1) with $E$ substituted by $U_{n A}$.

To be concrete we consider neutrons with energy $E=80 \mathrm{neV}$ which according to (11) corresponds to $v=3.9 \cdot 10^{2} \mathrm{~cm} / \mathrm{sec}$. Such neutrons have momenta $k \simeq 12.3 \mathrm{eV}$, de Broglie wave lengths $\lambda \simeq 10^{-5} \mathrm{~cm}$. As for the wall material we take $C^{12}$ with the density $\rho=2.25 \mathrm{~g} / \mathrm{cm}^{3}$, or $N \simeq 1.13$. $10^{-16} \mathrm{fm}^{-3}$. The coherent $n C^{12}$ scattering length is $a_{n}=6.65 \mathrm{fm}$ [ 8 ] (the 
imaginary part of the scattering length is at least 3 orders of magnitude smaller and is ignored as was already stated). The corresponding $n C^{12}$ optical potential reads

$$
U_{n}=\frac{2 \pi}{m} N a_{n} \simeq 195 n e V,
$$

with $m$ being the neutron mass. Limiting velocity corresponding to $E=U_{n}$ is $v_{n}^{(0)} \simeq 6.1 \cdot 10^{2} \mathrm{~cm} / \mathrm{sec}$. Thus neutrons under consideration with $v_{n}=3.9 \cdot 10^{2}$ $\mathrm{cm} / \mathrm{sec}$ are certainly ultra-cold with respect to $C^{12}$ trap walls.

The experimental data on antineutron-nuclei scattering lengths at ultralow energies are absent. Only some indirect information may be gained from the level shifts of antiprotonic atoms. The energy behavior of the $\bar{n} A$ annihilation cross-section is governed by the well-known " $1 / v$ " law

$$
\sigma_{a}=-4 \pi \frac{\operatorname{Ima_{\overline {n}A}}}{k}
$$

Several fits to the $\bar{n} A$ scattering lengths have been proposed in the literature. We consider as most reliable that of Ref. [9] based on internuclear cascade model. Even within this particular model one finds several solutions for $\bar{n} C^{12}$ scattering length. Therefore the one we have chosen for our analysis may be called "motivated" by Ref. [9] and reads

$$
a_{\bar{n}}=(3-i 1) f m .
$$

Then according to (2) the $\bar{n} C^{12}$ optical potential is equal to

$$
V_{\bar{n}}=U_{\bar{n}}-i W_{\bar{n}} \simeq(90-i 30) \mathrm{neV} .
$$

The limiting $\bar{n}$ velocity corresponding to $U_{\bar{n}}$ from (5) is according to (11) $v_{\bar{n}}^{(0)} \simeq 4.15 \cdot 10^{2} \mathrm{~cm} / \mathrm{sec}$. Now our choice $E=80 \mathrm{neV}<U_{\bar{n}}<U_{n}$ is clear since we want to deal with "ultra-cold" $\bar{n}$ as well. The case $U_{\bar{n}}<E<U_{n}$ will be considered in the next publication. Needless to say that due to strong $\bar{n}$ absorption the condition $E_{\bar{n}}<U_{\bar{n}}$ in no way provides complete reflection of $\bar{n}$ from the wall - see below.

Next we remind that the lower limit on $n-\bar{n}$ oscillation time $\tau_{n \bar{n}}$ has been obtained from experimental study of $n-\bar{n}$ transitions in free space and inside nuclei - see [10, 4, 11, 9] and references therein. For our purposes it is enough to keep in mind a crude value

$$
\tau_{n \bar{n}}>10^{8} \mathrm{sec}
$$


Correspondingly the value of the mixing parameter is

$$
\varepsilon_{n \bar{n}}=\frac{1}{\tau_{n \bar{n}}}<10^{-23} \mathrm{eV}
$$

The dynamical meaning of $\varepsilon_{n \bar{n}}$ will be clear from the evolution equation which will be presented below. To give some perception of the value of $\varepsilon_{n \bar{n}}$ we may say that if one considers neutron confined in a one-dimensional 5 meters long box then the level splitting will be just $10^{-23} \mathrm{eV}$.

Finally we introduce the parameter $t_{L} \sim 1 \mathrm{sec}$ - the time which neutrons need to cross the trapping box, or the time between two subsequent collisions with the walls. We also remind that both $\beta$-decay time and the UCN storage time are of the order of $10^{3} \mathrm{sec}$.

\section{$3 \quad$ Hitting the Trap Wall: Simple Estimates}

Now with relevant parameters at hands we can analyze what happens with neutrons and possible antineutrons admixture when they interacts with the wall of the trap. In this section we present simple estimates.

Treatment based on time evolution equation will be postponed till the next section. A third approach based on the wave packets formulation will be only touched on in present paper and discussed in detail in the next publication.

First we consider the collision of neutrons with the wall. As compared to antineutrons this problem is much simpler due to the lack of absorption (see remarks on the previous pages).

In our illustrative example a neutron with $v=3.9 \cdot 10^{2} \mathrm{~cm} / \mathrm{sec}$ is hitting the $C^{12}$ wall of the trap. Such a velocity is well below the limiting $C^{12}$ value $v_{n}^{(0)} \simeq 6.1 \cdot 10^{2} \mathrm{~cm} / \mathrm{sec}$ and hence neutron undergoes a complete reflection, $R=1$. Being absolutely correct, the statement that the reflection coefficient $R=1$ does not constitute the whole story. First, even at $v<v_{\bar{n}}^{(0)}$ the tail of the neutron wave function penetrates inside the wall. On general grounds the penetration depth is $l_{w}(n) \sim \lambda \simeq 10^{-5} \mathrm{~cm}$, with $\lambda$ being de Broglie wave length. Second, collision with the wall is not an instantaneous act but is characterized by certain collision time. The rigorous derivation of this time should be based on the wave packets formalism [12]-14]. However simple estimates presented below yield the same results. 
Inside the wall the neutron wave function has the form

$$
\psi_{\bar{n}}(x) \propto \exp \left\{-\kappa_{n} x\right\}, \quad \kappa_{n}=\sqrt{2 m\left(U_{n}-E\right)} .
$$

From (8) it is natural to identify

$$
l_{w}(n) \sim 1 / \kappa_{n} \simeq 0.14 \cdot 10^{-5} \mathrm{~cm},
$$

which is few times less than the naive expectation $l_{w}(n) \sim \lambda=10^{-5} \mathrm{~cm}$. Collision time may be estimated as凹

$$
\tau_{\text {coll }}(n) \sim \frac{2 l_{w}(n)}{v} \simeq 0.7 \cdot 10^{-8} \mathrm{sec} .
$$

This result is in perfect agreement with what is predicted from collision theory formulated in terms of the wave packets. Namely, if one describes the incident neutron by a wave packet

$$
\psi_{n}(x, t)=\sqrt{\frac{a}{\pi}} \exp \left(i k x-\frac{k^{2} t}{2 m}\right) \frac{\sin [(x-v t) / a]}{x-v t},
$$

where $a$ is its width in $x$-space, then

$$
\tau_{\text {coll }}=\left[E\left(U_{0}-E\right)\right]^{-1 / 2} \simeq 0.7 \cdot 10^{-8} \mathrm{sec}
$$

for $E=80 \mathrm{neV}, U_{0}=195 \mathrm{neV}$. At this point we note that $\tau_{\text {coll }} v=2 l_{w} \ll$ $a=\frac{\lambda}{\pi}\left(\frac{\Delta \lambda}{\lambda}\right)^{-1}$. We shall return to this remark in connection with possible decoherence of $n$ and $\bar{n}$ due to the difference in their collision times.

Now we turn to $\bar{n}$ with the same velocity $v=3.9 \cdot 10^{2} \mathrm{~cm} / \mathrm{sec}$ hitting the $C^{12}$ wall.

Due to absorption (annihilation) the $\bar{n}$ wave function inside the wall has the form

$$
\begin{gathered}
\psi_{\bar{n}}(x) \propto e^{i k_{\bar{n}} x-\kappa_{\bar{n}} x}, \\
\left(i k_{\bar{n}}-\kappa_{\bar{n}}\right)^{2}=2 m\left(U_{\bar{n}}-i W_{\bar{n}}-E\right),
\end{gathered}
$$

where $E$ is the energy of the incident $\bar{n}$. Eqs. (13) 14) yield

$$
l_{w}(\bar{n}) \simeq \frac{1}{\kappa_{\bar{n}}}=m^{-1 / 2}\left\{U_{\bar{n}}-E+\left[\left(U_{\bar{n}}-E\right)^{2}+W^{2}\right]^{1 / 2}\right\}^{-1 / 2} \simeq 0.32 \cdot 10^{-5} \mathrm{~cm} .
$$

\footnotetext{
${ }^{1}$ Strictly speaking neutron velocity inside the wall is different from $v$.
} 
Then $\bar{n}$ collision time is

$$
\tau_{\text {coll }}(\bar{n}) \sim \frac{2 l_{w}(\bar{n})}{v} \simeq 1.6 \cdot 10^{-8} \mathrm{sec},
$$

which is about 2 times larger than the neutron collision time given by (10)?

The crucial parameter which determines the fate of $\bar{n}$ hitting the wall is the ratio of the collision time (16) to the absorption (annihilation) time. The later quantity is velocity independent by virtue of the " $1 / v$ " law (3) and is expressed through the $\bar{n}$ mean free path $\Lambda$ according to

$$
\tau_{a b s}(\bar{n}) \sim \frac{\Lambda}{v} \simeq \frac{m}{4 \pi N\left|I m a_{\bar{n} A}\right|} \simeq 1.1 \cdot 10^{-8} \mathrm{sec}
$$

for the $C^{12}$ trap wall. Thus

$$
\tau_{\text {coll }}(\bar{n}) / \tau_{a b s}(\bar{n})>1,
$$

which implies the collapse of the possible $\bar{n}$ component on the wall.

Already at this point it is clear that this in turn leads to the time dependence of the probability to find $\bar{n}$ component announced in the Abstract, namely $P(\bar{n})=\varepsilon^{2} t_{L} t$. A rigorous derivation of this equation is given in the next section.

Still one may argue that the above estimates should be taken with caution and certain fraction of $\bar{n}$ may be still reflected from the wall. Then Eqs.(10) and (16) enable to estimate the splitting between the centers of the $n$ and $\bar{n}$ wave packets (see (11)) after the reflection. One has

$$
\delta x \simeq v\left(\tau_{\text {coll }}(\bar{n})-\tau_{\text {coll }}(n)\right) \simeq 0.35 \cdot 10^{-5} \mathrm{~cm} \sim \lambda \ll a .
$$

Whether this retardation influences the $n-\bar{n}$ mixing in free space between collisions with the trap walls will be discussed elsewhere.

The main point to be improved on in the above estimates is mentioned in the footnote to Eq.(10). Certain guidance in this direction may be found in 15 .

Finally we note that the treatment presented above seems physically more transparent than formal calculations of the reflection coefficient from the complex potential.

\footnotetext{
${ }^{2}$ Wave packets formalism, as was shown by V.A.Lensky, leads to somewhat smaller value - see our next publication.
} 


\section{Hitting the Trap Wall: Time-Dependent Approach}

As a "warming up" exercise we consider $n-\bar{n}$ oscillations in a free space with $\beta$ - decay neglected. This is a standard two-level problem treated in any serious textbook on Quantum Mechanics. The phenomenological Hamiltonian is a $2 \times 2$ matrix in the basis of the two-component $n-\bar{n}$ wave function

$$
H=E_{i} \delta_{i j}+\varepsilon \sigma_{x}
$$

with $i, j=n, \bar{n}$. The evolution equation reads

$$
i \frac{\partial}{\partial t}\left(\begin{array}{l}
\psi_{n} \\
\psi_{\bar{n}}
\end{array}\right)=\left(\begin{array}{ll}
E_{n} & \varepsilon \\
\varepsilon & E_{\bar{n}}
\end{array}\right)\left(\begin{array}{l}
\psi_{n} \\
\psi_{\bar{n}}
\end{array}\right) .
$$

Assuming that $\psi_{n}(t=0)=1, \quad \psi_{\bar{n}}(t=0)=0$, and diagonalizing the Hamiltonian (20) one arrives at the following expression for the probability of finding $\bar{n}$ at a time $t$ [2]- 6 ]

$$
\left.\psi_{\bar{n}}(t)\right|^{2}=\frac{4 \varepsilon^{2}}{\omega^{2}+4 \varepsilon^{2}} \sin ^{2}\left(\frac{1}{2} \sqrt{\omega^{2}+4 \varepsilon^{2}} t\right),
$$

where $\omega=\left(E_{\bar{n}}-E_{n}\right)$. In free space the difference between $E_{\bar{n}}$ and $E_{n}$ may be due to the Earth magnetic field. In this case

$$
\omega=2 \mu_{n} B \simeq 6 \cdot 10^{-12} \mathrm{eV} .
$$

Without magnetic field, i.e. at $\omega=0$, and at $t \ll \tau_{n \bar{n}} \simeq 10^{8}$ sec one has

$$
\left|\psi_{\bar{n}}(t)\right|^{2} \simeq \varepsilon_{n \bar{n}}^{2} t^{2}
$$

while with the Earth magnetic field Eq.(24) is valid only at extremely short times, $t \ll(\mu B)^{-1} \simeq 2 \cdot 10^{-4}$ sec, while at large times

$$
\left|\psi_{\bar{n}}(t)\right|^{2} \simeq \frac{4 \varepsilon^{2}}{\omega^{2}} \sin ^{2} t / \tau_{B} \simeq 10^{-23} \sin ^{2} t / \tau_{B}
$$

where $\tau_{B}=(\mu B)^{-1} \simeq 2 \cdot 10^{-4}$ sec.

The use of (22) to test fundamental symmetries is discussed in [5].

Next we consider the general Hamiltonian of the $n-\bar{n}$ system inside the wall with annihilation and $\beta$-decay included. The problem is reminiscent 
of strangeness oscillations in $K \bar{K}$ system. With annihilation and $\beta$-decay included the Hamiltonian (20) is substituted by

$$
H=\left(\begin{array}{ll}
E_{n}-i \frac{\Gamma_{\beta}}{2} & \varepsilon \\
\varepsilon & E_{\bar{n}}-i \frac{\Gamma_{a}}{2}-i \frac{\Gamma_{\beta}}{2}
\end{array}\right),
$$

where $\Gamma_{\beta}^{-1} \sim 10^{3} \mathrm{sec}, \Gamma_{a} \simeq 2 W_{n} \simeq 60 \mathrm{neV}$ for $C^{12}$.

In arriving to (22) diagonalization of the Hamiltonian (20) has been done exactly. Performing similar procedure with (26) use can be made of a small parameter $4 \varepsilon^{2} \ll\left|H_{11}-H_{22}\right|^{2}$. Indeed, inside the wall effective fields acting on $n$ and $\bar{n}$ differ by tens of neV (see (2) and (5)) while $\varepsilon \sim 10^{-14} \mathrm{neV}$. Expanding $\left\{\left(H_{11}-H_{22}\right)^{2}+4 \varepsilon^{2}\right\}^{1 / 2}$ with respect to this small parameter one finds the two eigenvalues of the Hamiltonian (26)

$$
\begin{gathered}
\mu_{1} \simeq E_{n}^{\prime}-i \frac{\Gamma_{\beta}}{2}-i \frac{\Gamma_{\varepsilon}}{2}, \\
\mu_{2} \simeq E_{\bar{n}}^{\prime}-i \frac{\Gamma_{a}}{2}-i \frac{\Gamma_{\beta}}{2}+i \frac{\Gamma_{\varepsilon}}{2} .
\end{gathered}
$$

Here $E_{n}^{\prime}=E_{n}-E_{\varepsilon}, \quad E_{\bar{n}}^{\prime}=E_{\bar{n}}+E_{\varepsilon}$, and

$$
E_{\varepsilon}+i \frac{\Gamma_{\varepsilon}}{2}=\frac{\varepsilon^{2}}{E_{\bar{n}}-E_{n}-i \frac{\Gamma_{a}}{2}} .
$$

The "wrong" sign of the last term in (28) is an artifact of the square root expansion, but this is physically irrelevant since $\Gamma_{\varepsilon} \ll \Gamma_{\beta} \ll \Gamma_{a}\left(\Gamma_{\varepsilon} \sim\right.$ $\left.10^{-39} \mathrm{eV}, \quad \Gamma_{\beta} \sim 10^{-18} \mathrm{eV}, \quad \Gamma_{a} \sim 10^{-7} \mathrm{eV}\right)$.

In terms of eigenvalues $\mu_{1}$ and $\mu_{2}$ the general solution of the two-component evolution equation has the form [16]

$$
\psi(t)=\left(\begin{array}{c}
\psi_{n}(t) \\
\psi_{\bar{n}}(t)
\end{array}\right)=\left(\frac{H-\mu_{2}}{\mu_{1}-\mu_{2}} e^{-i \mu_{1} t}+\frac{H-\mu_{1}}{\mu_{2}-\mu_{1}} e^{-i \mu_{2} t}\right) \psi(0) .
$$

Again we start with a solution corresponding to initial conditions $\psi_{n}(t=$ $0)=1, \quad \psi_{\bar{n}}(t=0)=0$. Then from $(26)-(30)$ one gets

$$
\left|\psi_{\bar{n}}(t)\right|^{2}=\frac{\varepsilon^{2}}{\omega^{2}+\frac{\Gamma_{a}^{2}}{4}} e^{-\left(\Gamma_{\beta}+\Gamma_{\varepsilon}\right) t}\left\{1+e^{-\Gamma_{a}^{\prime} t}-2 e^{-\frac{\Gamma_{a}^{\prime}}{2} t} \cos \omega t\right\},
$$


where $\omega=\left(E_{\bar{n}}-E_{n}\right), \quad \Gamma_{a}^{\prime}=\Gamma_{a}-2 \Gamma_{\varepsilon}$, and $\Gamma_{\varepsilon}$ is defined by (29). Since $\Gamma_{\varepsilon} \ll \Gamma_{\beta}$ and $\Gamma_{\varepsilon} \ll \Gamma_{a}$, one can rewrite (31) in a simpler form without noticeable lost of accuracy, namely

$$
\left|\psi_{\bar{n}}(t)\right|^{2}=\frac{\varepsilon^{2}}{\omega^{2}+\frac{\Gamma_{a}^{2}}{4}} e^{-\Gamma_{\beta} t}\left\{1+e^{-\Gamma_{a} t}-2 e^{-\frac{\Gamma_{a}}{2} t} \cos \omega t\right\}
$$

This equation resembles that giving the probability to find $\bar{K}^{0}$ in initially pure $K^{0}$ beam. Notice that instead of the overall factor $1 / 4$ for $K^{0}-\bar{K}^{0}$ system we find in (32) an extremely small factor $\varepsilon^{2}\left(\omega^{2}+\Gamma_{a}^{2} / 4\right)^{-1} \sim 10^{-32}($ !) reflecting the fact that mixing is very small as compared to the complex splitting of $n$ and $\bar{n}$ eigenvalues in the medium.

Consider (32) at $t=\tau_{\text {coll }}(\bar{n}) \simeq 1.6 \cdot 10^{-8} \sec ($ see $(16))$. Then $\Gamma_{\beta} \tau_{\text {coll }}(\bar{n}) \sim$ $10^{-11}, \quad \Gamma_{a} \tau_{\text {coll }}(\bar{n}) \simeq 1.5, \quad \cos \omega \tau_{\text {coll }}(\bar{n}) \simeq 0.7$, and (32) yields

$$
\left|\psi_{\bar{n}}\left(\tau_{\text {coll }}(\bar{n})\right)\right|^{2} \lesssim 10^{-32}
$$

Physically this means that if a pure $n$ beam collides with a wall made of $C^{12}$ the tiny admixture of $\bar{n}$ which would have emerged during the collision time is completely damped by annihilation and $n-\bar{n}$ energy splitting.

The free-space regime (24) is "hidden" in (32) at the limit of very short times, $t \ll 1 / \Gamma_{a} \sim 10^{-8}$ sec. Then

$$
\left|\psi_{\bar{n}}(t)\right|^{2} \simeq \frac{\varepsilon^{2}}{\omega^{2}+\frac{\Gamma_{a}^{2}}{4}} \sin ^{2} \frac{\omega t}{2} \simeq \frac{\varepsilon^{2} t^{2}}{1+\Gamma_{a}^{2} / 4 \omega^{2}} \simeq 0.9 \varepsilon^{2} t^{2},
$$

where at the last step use has been made of the values of $\Gamma_{a}$ and $\omega$ for $C^{12}$.

Next consider (30) at initial conditions which are closer to real experimental situation. Namely, suppose that UCN beam collides with the wall after crossing the trap. The Earth magnetic field is assumed to be shielded, so that the free-space equation (24) is valid inside the trap. Then initial conditions in (30) read

$$
\psi_{\bar{n}}(t=0)=\varepsilon t_{L}, \quad \psi_{n}(t=0)=\sqrt{1-\varepsilon^{2} t_{L}^{2}},
$$

where $t_{L} \simeq 1 \mathrm{sec}$. Then at $t=\tau_{\text {coll }}(\bar{n}) \simeq 1.6 \cdot 10^{-8}$ sec, i.e. just after the collision with the wall, one gets

$$
\left|\psi_{\bar{n}}\left(\tau_{\text {coll }}\right)\right|^{2} \simeq \varepsilon^{2} t_{L}^{2} e^{-\left(\Gamma_{a}+\Gamma_{\beta}\right) \tau_{c o l l}}\left[1+0\left(1 / t_{L} \Gamma_{a}\right)\right]
$$


This result is again physically transparent. During the collision the $\bar{n}$ component is depleted by annihilation, while antineutrons "newly born" inside the wall are damped according to our previous result (32).

Now $\tau_{\text {coll }}(\bar{n}) \simeq 1.6 \cdot 10^{-8} \mathrm{sec}, \Gamma_{a} \simeq 60 \mathrm{NeV}$, so that $\exp \left(-\Gamma_{a} \tau_{\text {coll }}\right) \simeq 0.2$. This means that only 1 per $\simeq 5 \bar{n}$ survives after the collision.

This result is in line with estimates presented in Section 3 but here we are on somewhat more qualitative footing.

If one considers the fraction $1 / 5$ as a small parameter, then the probability of antineutron detection at time $t$ will be

$$
\left|\psi_{\bar{n}}(t)\right|^{2}=\varepsilon^{2} t_{L} t
$$

instead of $\varepsilon^{2} t^{2}$ free-space law (24). Indeed, the probability of $n-\bar{n}$ transition between the two subsequent collisions with the walls is $\varepsilon^{2} t_{L}^{2}$, while the number of collisions during the observation time is $\left(t / t_{L}\right)$. The extrapolation between the laws (24) and (37) will be discussed in the next publication.

\section{Acknowledgements}

The author is deeply grateful to Yu.A.Kamyshkov for grabbing him into the subject and presenting a clear introduction. Discussion of the oscillation problem with M.I.Vysotsky was very enlightening. Useful remarks and information was gained from V.A.Lensky, L.A.Kondratyuk, A.E.Kudryavtsev and the members of the ITEP seminar.

Special thanks for essential financial support are to V.A.Novikov, L.B.Okun and grants RFFI 00-02017836 and 00-15-96786.

\section{References}

[1] V.A. Kuzmin, JETP Lett. 13, 335 (1970).

[2] K.G. Chetyrkin et.al., Phys. Lett. B 99, 358 (1970).

[3] M.Baldo Ceolin, In: Festischrift for Val Telegdi, ed. K. Winter. Elsevier Publ., Amsterdam 1988, p.17.

[4] A.Gal, Phys. Rev. C 61, 028201 (2000). 
[5] Yu.G.Abov, F.S.Dzheparov and L.B.Okun, Pis'ma v ZheTF, 39, 493 (1984).

[6] R.Golub, In: Proc. of the Int. Workshop on Future Prospects of Baryon Instability Search, ed. S.J.Ball and Y.A.Kamyshkov, Oak Ridge Nat. Lab. , 1996, p. 329.

[7] Ya.B. Zeldovich, ZheTF, 36, 1952 (1959).

[8] V.K.Ignatovich, The Physics of Ultra-Cold Neutrons, "Nauka", Moscow, 1986, p. 228 (in Russian).

[9] Ye.S. Golubeva and L.A. Kondratyuk, Nucl. Phys. B (Proc.Suppl.) 56 A, 103 (1997).

[10] M.Baldo Ceolin et.al., Zeit. fur Phys. C63, 409 (1994).

[11] J.Chung et.al., Phys. Rev. D66, 032004 (2002).

[12] A.Messiah, Quantum Mechanics vol. 1, Amsterdam, North-Holland, 1962.

[13] M.L.Goldberger and K.M.Watson, Collision Theory, John Wiley, New York-London-Sydney, 1964.

[14] V.M.Galitsky, B.M.Karnakov and V.I.Kogan, Problems in Quantum Mechanics, " Nauka", Moscow, 1992 (in Russian).

[15] I.M.Frank, Uspekhi Fizicheskikh Nauk 161, 109 (1991).

[16] I.Yu.Kobzarev, N.N.Nikolaev and L.B.Okun, Yad. Fiz. 10, 864 (1969). 